\title{
Enumeration of 3- and 4-Wilf classes of four 4-letter patterns
}

\author{
David Callan $^{1}$ and Toufik Mansour ${ }^{2}$ \\ ${ }^{1}$ Department of Statistics, University of Wisconsin \\ Madison, WI 53706, United States \\ e-mail: callanestat.wisc.edu \\ 2 Department of Mathematics, University of Haifa \\ 3498838 Haifa, Israel \\ e-mail: tmansoureuniv.haifa.ac.il
}

\begin{abstract}
Let $S_{n}$ be the symmetric group of all permutations of $n$ letters. We show that there are precisely 27 (respectively, 15) Wilf classes consisting of exactly 3 (respectively, 4) symmetry classes of subsets of four 4-letter patterns.
\end{abstract}

Keywords: Pattern avoidance, Wilf-equivalence.

2010 Mathematics Subject Classification: 05A15, 05A05.

\section{Introduction}

This paper is a sequel to [2] (also, see [1]) and continues the investigaton of permutations avoiding a quadruple of (distinct) 4-letter patterns. In [2] we determined all 64 Wilf classes consisting of exactly 2 symmetry classes of quadruples of 4-letter patterns. Following the same terminology and notation as in that paper, here we establish the following results.

Theorem 1. The number of Wilf classes consisting of exactly 3 symmetry classes of subsets of 4 patterns in $S_{4}$ (3-Wilf classes) is 27.

Theorem 2. The number of Wilf classes consisting of exactly 4 symmetry classes of subsets of 4 patterns in $S_{4}$ (4-Wilf classes) is 15 . 
For Theorem 1, a perusal of the counting sequences $\left(\left|S_{n}(T)\right|\right)_{n=1, \ldots, 16}$ for a representative quadruple $T$ in each symmetry class of 4 patterns in $S_{4}$ shows that there are at most 273 -Wilf classes of subsets of 4 patterns in $S_{4}$, see Table 1 in the appendix below. We used the insertion encoding algorithm (INSENC) [7] on the symmetry classes in Table 1 and successful outcomes, always a rational generating function, are referenced by "INSENC". To prove Theorem 1, we find in Section 2 an explicit formula for the generating function $F_{T}(x)=\sum_{n \geq 0}\left|S_{n}(T)\right| x^{n}$ for each $T$ that appears in one of the 27 candidate triples, whenever $F_{T}(x)$ is nonrational. The 13 cases where $F_{T}(x)$ is rational and INSENC fails are marked "EX" for Exercise in Table 1, and their proofs are omitted.

The analogous results for Theorem 2 are listed in Table 2 and we include a selection of proofs in Section 3.

\section{Proof of Theorem 1}

\subsection{Case 656}

The enumeration of the first two symmetry classes is obtained from [4]. Thus it remains to enumerate the last class $T=\{2341,2314,1243,1234\}$. Define $a_{n}\left(i_{1}, i_{2}, \ldots, i_{s}\right)$ to be the number of permutations $\pi=i_{1} i_{2} \cdots i_{s} \pi^{\prime} \in S_{n}(T)$. Define $A_{n}(v)=\sum_{i=1}^{n} a_{n}(i) v^{i-1}$ and $A(x, v)=$ $\sum_{n \geq 0} A_{n}(v) x^{n}$. To find an explicit formula for $A(x, v)$, we define $a_{n}^{+}(i)=\sum_{j=i+1}^{n} a_{n}(i, j)$, $A_{n}^{+}(v)=\sum_{i=1}^{n-1} a_{n}^{+}(i) v^{i-1}$ and $A^{+}(x, v)=\sum_{n \geq 2} A_{n}^{+}(v) x^{n}$.

Lemma 3. We have

$$
A^{+}(x, v)=\frac{x^{3}}{1-2 x}+x(A(x, v)-1) .
$$

Proof. Since we avoid 2314 and 2341 we have $a_{n}(i, j)=0$ for all $1 \leq i<j \leq n-1$ such that $(i, j) \neq(1, n-1)$. We have $\left|S_{n}(123,132)\right|=2^{n-1}$ [6], so $a_{n}(1, n-1)=2^{n-3}$. Moreover, from the definitions, $a_{n}(i, n)=a_{n-1}(i)$. Therefore,

$$
a_{n}^{+}(i)=2^{n-3} \delta_{i=1}+a_{n-1}(i)
$$

Multiplying by $v^{i-1}$ and summing over $i=1,2, \ldots, n$, we obtain for $n \geq 3$,

$$
A_{n}^{+}(v)=2^{n-3}+A_{n-1}(v)
$$

with $A_{2}^{+}(v)=1$, and the result follows by summing over $n \geq 2$.

Similarly, we define $a_{n}^{-}(i)=\sum_{j=1}^{i-1} a_{n}(i, j), A_{n}^{-}(v)=\sum_{i=1}^{n} a_{n}^{-}(i) v^{i-1}$ and $A^{-}(x, v)=$ $\sum_{n \geq 2} A_{n}^{-}(v) x^{n}$.

Lemma 4. With $v=1 / C(x)$, we have

$$
A^{-}(x, 1)=\left(v^{2} x-v^{2}+v x-x+1\right) A(x, 1)-\frac{v^{3} x-v^{2} x^{2}-v^{3}+2 v^{2} x-x^{3}+v-2 x}{v-2 x} .
$$


Proof. For $2 \leq j<i \leq n-2$, if $\pi=i j \pi^{\prime} \in S_{n}(T)$, then the leftmost letter of $\pi^{\prime}$ is either smaller than $j$ or equal to $n$ (the maximal letter), and so

$$
a_{n}(i, j)=\sum_{k=1}^{j-1} a_{n}(i, j, k)+a_{n}(i, j, k) .
$$

Note that $\pi=i j(j-1) \pi^{\prime}$ avoids $T$ if and only if $(i-1)(j-1) \pi^{\prime}$ avoids $T$, and for $k \leq j-2$, $\pi=i j k \pi^{\prime}$ avoids $T$ if and only if $j k \pi^{\prime}$ avoids $T$, and $\pi=i j n \pi^{\prime}$ avoids $T$ if and only if $i j \pi^{\prime}$ avoids $T$. Hence, for $2 \leq j<i \leq n-2$,

$$
a_{n}(i, j)=\sum_{k=1}^{j-2} a_{n-1}(j, k)+a_{n-1}(i-1, j-1)+a_{n-1}(i, j),
$$

which is equivalent to

$$
a_{n}(i, j)=a_{n-1}^{-}(j)-a_{n-2}(j-1)+a_{n-1}(i-1, j-1)+a_{n-1}(i, j) .
$$

Summing over $j=2,3, \ldots, i-2$ and using the fact $a_{n}(i, i-1)=a_{n-1}(i-1)$, we have

$$
\begin{aligned}
a_{n}^{-}(i) & -a_{n}(i, 1)-a_{n-1}(i-1) \\
& =\sum_{j=2}^{i-2} a_{n-1}^{-}(j)-\sum_{j=1}^{i-3} a_{n-2}(j) \\
& +a_{n-1}^{-}(i-1)-a_{n-2}(i-2)+a_{n-1}^{-}(i)-a_{n-1}(i, 1)-a_{n-2}(i-1) .
\end{aligned}
$$

Note that $a_{n}^{-}(1)=0, a_{n}^{-}(n)=a_{n-1}$ and $a_{n}^{-}(n-1)=a_{n-1}-a_{n-2}$. Also, $a_{n}(i, 1)=2^{i-2}$ since $\left|S_{n}(123,132)\right|=2^{n-1}$. Hence,

$$
a_{n}^{-}(i)=\sum_{j=1}^{i} a_{n-1}^{-}(j)-\sum_{j=1}^{i-1} a_{n-2}(j)+a_{n-1}(i-1)
$$

with $a_{n}^{-}(n)=a_{n-1}$ and $a_{n}^{-}(n-1)=a_{n-1}-a_{n-2}$.

Multiplying the last recurrence by $v^{i-1}$ and summing over $i=1,2, \ldots, n-2$, we obtain

$$
\begin{aligned}
A_{n}^{-}(v) & =\frac{1}{1-v}\left(A_{n-1}^{-}(v)-v^{n-2} A_{n-1}^{-}(1)\right)-\frac{v}{1-v}\left(A_{n-2}(v)-v^{n-1} A_{n-2}(1)\right) \\
& +v A_{n-1}(v)+v^{n-2}(1+v) A_{n-1}(1)-v^{n-2} A_{n-2}(1)
\end{aligned}
$$

with $A_{2}^{-}(v)=v$. Hence, multiplying by $x^{n}$ and summing over $n \geq 3$, we get

$$
\begin{aligned}
A^{-}(x, v) & =\frac{x}{1-v}\left(A^{-}(x, v)-\frac{1}{v} A^{-}(v x, 1)\right)-\frac{v x^{2}}{1-v}(A(x, v)-v A(v x, 1)) \\
& +v x(A(x, v)-1)+x(A(v x, 1)-1)+\frac{x}{v}(A(v x, 1)-1)-x^{2} A(v x, 1) .
\end{aligned}
$$

By Lemma 3, we have

$$
A(x, v)=1+x+A^{+}(x, v)+A^{-}(x, v)=1+x+\frac{x^{3}}{1-2 x}+x(A(x, v)-1)+A^{-}(x, v),
$$


which leads to

$$
A(x, v)=\frac{1-x-x^{2}}{1-2 x}+\frac{1}{1-x} A^{-}(x, v) .
$$

Lemma 3 also gives

$$
A^{+}(v x, 1)=\frac{v^{3} x^{3}}{1-2 v x}+v x(A(v x, 1)-1),
$$

Hence, by plugging the expressions for $A(x, v)$ and $A^{+}(v x, 1)$ into (1), we obtain

$$
\begin{aligned}
\frac{\left(v^{2}-v+x\right)(v x-v+x)}{v(1-v)(v-x)} & A^{-}(x / v, v) \\
& =\frac{x}{v^{2}(1-v)} A^{-}(x, 1)-\frac{x\left(v^{2} x-v^{2}+v x-x+1\right)}{v^{2}(1-v)} A(x, 1) \\
& +\frac{x\left(v^{3} x-v^{2} x^{2}-v^{3}+2 v^{2} x-x^{3}+v-2 x\right)}{v^{2}(v-2 x) /(1-v)}
\end{aligned}
$$

By taking $v=1 / C(x)$, we complete the proof.

Since $A(x, 1)=1+x+A^{-}(x, 1)+A^{+}(x, 1)$, Lemmas 3 and 4 imply the following result.

Theorem 5. Let $T=\{2341,2314,1243,1234\}$. Then

$$
F_{T}(x)=C(x)+\frac{x^{3}}{1-2 x} C(x)^{4} .
$$

\subsection{Case 890}

\subsubsection{The symmetry class of $\{2143,1243,1423,1432\}$}

Let $a_{n}=\left|S_{n}(T)\right|$ with $T=\{2143,1243,1423,1432\}$. Define $a_{n}\left(i_{1}, i_{2}, \ldots, i_{s}\right)$ to be the number of permutations $\pi=i_{1} i_{2} \cdots i_{s} \pi^{\prime} \in S_{n}(T)$, and set $b_{n}(i)=a_{n}(i, i+1)$ and $c_{n}(i)=a_{n}(i, i+2)$.

Lemma 6. For $1 \leq i \leq n-2, a_{n}(i, j)=0$ whenever $j>i+3, c_{n}(i)=a_{n-1}(i), b_{n}(i)=$ $a_{n-1}(i)-c_{n-1}(i)$, and

$$
a_{n}(i)=c_{n}(1)+c_{n}(2)+\cdots+c_{n}(i-2)+b_{n}(i-1)+b_{n}(i)+c_{n}(i),
$$

while $a_{n}(n)=a_{n}(n-1)=a_{n-1}$.

Proof. For $\pi=i j \pi^{\prime} \in S_{n}$ with $i+3 \leq j, \pi$ has an occurrence of either 1423 or 1432 . Thus $a_{n}(i, j)=0$ whenever $j>i+3$. From now we assume that $1 \leq i \leq n-2$. First, suppose $\pi=i(i+2) j \pi^{\prime} \in S_{n}(T)$. Then either $j \leq i-1$ or $j=i+1$ or $j=i+3$. Thus

$$
c_{n}(i)=\sum_{j=1}^{i+2} a_{n-1}(i, j)=a_{n-1}(i) .
$$

Next, let $\pi=i(i+1) j \pi^{\prime} \in S_{n}(T)$. Then either $j \leq i-1$ or $j=i+2$. Thus

$$
b_{n}(i)=\sum_{j=1}^{i+1} a_{n-1}(i, j)=a_{n-1}(i)-c_{n-1}(i) .
$$


Now, let $\pi=i j \pi^{\prime} \in S_{n}(T)$. If $j=i-1$, then by exchanging the position of the letters $i$ and $i-1$, we see that $a_{n}(i, i-1)=a_{n}(i-1, i)=b_{n}(i-1)$ for all $i=2,3, \ldots, n$. If $j=i-2$, then

$$
a_{n}(i, i-2)=\sum_{k=1}^{i-3} a_{n}(i, i-2, k)+a_{n}(i, i-2, i-1)+a_{n}(i, i-2, i+1),
$$

which leads to

$a_{n}(i, i-2)=\sum_{k=1}^{i-3} a_{n-1}(i-2, k)+a_{n-1}(i-2, i-1)+a_{n-1}(i-2, i)=a_{n-1}(i-2)=c_{n}(i-2)$.

So, let us assume that $1 \leq j \leq i-3$. Then

$$
a_{n}(i, j)=\sum_{k=1}^{j-1} a_{n}(i, j, k)+\sum_{k=j+1}^{j+2} a_{n}(i, j, k),
$$

which gives

$$
a_{n}(i, j)=\sum_{k=1}^{j-1} a_{n-1}(j, k)+\sum_{k=j+1}^{j+2} a_{n-1}(j, k)=a_{n-1}(j)=c_{n}(j)
$$

Hence,

$$
a_{n}(i)=c_{n}(1)+\cdots+c_{n}(i-2)+b_{n}(i-1)+b_{n}(i)+c_{n}(i)
$$

as required.

By Lemma 6 we have for $n \geq 3$,

$$
a_{n}(i)=a_{n-1}(1)+\cdots+a_{n-1}(i)+a_{n-1}(i)-a_{n-2}(i-1)-a_{n-2}(i) \delta_{i<n-2},
$$

and $a_{n}(n)=a_{n}(n-1)=a_{n-1}$. Define $A_{n}(v)=\sum_{i=1}^{n} a_{n}(i) v^{i-1}$. Then

$$
\begin{aligned}
A_{n}(v) & =\frac{1}{1-v}\left(A_{n-1}(v)-v^{n} A_{n-1}(1)\right) \\
& +A_{n-1}(v)-v^{n-2} A_{n-2}(1)-(1+v)\left(A_{n-2}(v)-v^{n-3} A_{n-3}(1)\right) .
\end{aligned}
$$

with $A_{0}(v)=A_{1}(v)=1$ and $A_{2}(v)=1+v$. Define $A(x, v)=\sum_{n \geq 0} A_{n}(v) x^{n}$, then by multiplying the last recurrence by $x^{n}$ and summing over $n \geq 3$, we obtain

$$
\begin{aligned}
A(x, v) & =1-x+(1+v) x^{2}+\frac{x}{1-v}(A(x, v)-v A(v x, 1)) \\
& +x(1-(1+v) x) A(x, v)-x^{2}(1-(1+v) x) A(v x, 1),
\end{aligned}
$$

which is equivalent to

$$
\begin{aligned}
& \left(1-\frac{x}{v(1-v)}-\frac{x}{v^{2}}(v-(1+v) x)\right) A\left(\frac{x}{v}, v\right) \\
& =1-\frac{x}{v}+(1+v) \frac{x^{2}}{v^{2}}-x\left(\frac{1}{1-v}+\frac{x}{v^{3}}(v-(1+v) x)\right) A(x, 1) .
\end{aligned}
$$

By taking $v$ as the root of $v=1+x-x^{2}-2 x / v+x^{2} / v^{2}$, we obtain the following result.

Theorem 7. Let $T=\{2143,1243,1423,1432\}$. Then

$$
F_{T}(x)=\frac{v\left(v(v-x)+(1+v) x^{2}\right)(1-v)}{x(v-x)},
$$

where $v=1-x-2 x^{2}-4 x^{3}-11 x^{3}-\cdots$ is the root of $v=1+x-x^{2}-2 x / v+x^{2} / v^{2}$. 


\subsubsection{The symmetry class of $\{2413,1423,1432,1342\}$}

Let $a_{n}=\left|S_{n}(T)\right|$ with $T=\{2413,1423,1432,1342\}$. Define $a_{n}\left(i_{1}, i_{2}, \ldots, i_{s}\right)$ to be the number of permutations $\pi=i_{1} i_{2} \cdots i_{s} \pi^{\prime} \in S_{n}(T)$. By similar techniques as in the proof of Lemma 6 , we obtain

Lemma 8. Define $b_{n}(i)=a_{n}(i, i+1)$ and $c_{n}(i)=a_{n}(i, i+2)$. For all $1 \leq i \leq n-2$, $a_{n}(i, j)=0$ whenever $j>i+3, c_{n}(i)=a_{n-2}(i)$ with $c_{n}(n-2)=a_{n-3}, b_{n}(i)=a_{n-1}(i)$ with $b_{n}(n-1)=a_{n-2}$, and

$$
a_{n}(i)=b_{n}(1)+\cdots+b_{n}(i)+c_{n}(i),
$$

and $a_{n}(n)=a_{n}(n-1)=a_{n-1}$.

Lemma 8 shows

$$
a_{n}(i)=a_{n-1}(1)+\cdots+a_{n-1}(i)+a_{n-2}(i),
$$

with $a_{n}(n)=a_{n}(n-1)=a_{n-1}$ and $a_{n}(n-2)=a_{n-1}-a_{n-2}+a_{n-3}$. Defining $A_{n}(v)=$ $\sum_{i=1}^{n} a_{n}(i) v^{i-1}$ and $A(x, v)=\sum_{n \geq 0} A_{n}(v) x^{n}$, we obtain

$$
A_{n}(v)=\frac{1}{1-v}\left(A_{n-1}(v)-v^{n} A_{n-1}(1)\right)+A_{n-2}(v)
$$

with $A_{0}(v)=A_{1}(v)=1$ and $A_{2}(v)=1+v$. So

$$
A(x / v, v)=1-\frac{x^{2}}{v^{2}}+\frac{x}{v(1-v)}(A(x / v, v)-v A(x, 1))+\frac{x^{2}}{v^{2}} A(x / v, v) .
$$

Hence, we can state the following result.

Theorem 9. Let $T=\{2413,1423,1432,1342\}$. Then

$$
F_{T}(x)=\frac{v\left(v(v-x)+(1+v) x^{2}\right)(1-v)}{x(v-x)},
$$

where $v=1-x-2 x^{2}-4 x^{3}-11 x^{3}-\cdots$ is the root of $v=1+x-x^{2}-2 x / v+x^{2} / v^{2}$.

\subsubsection{The symmetry class of $\{2314,1324,1243,1234\}$}

Let $a_{n}=\left|S_{n}(T)\right|$ with $T=\{2314,1324,1243,1234\}$. Define $a_{n}\left(i_{1}, i_{2}, \ldots, i_{s}\right)$ to be the number of permutations $\pi=i_{1} i_{2} \cdots i_{s} \pi^{\prime} \in S_{n}(T)$. By similar techniques as in the proof of Lemma 6 , we obtain

Lemma 10. Define $b_{n}(i)=a_{n}(i, n)$ and $c_{n}(i)=a_{n}(i, n-1)$. For all $1 \leq i \leq n-2, a_{n}(i, j)=0$ whenever $i+1 \leq j \leq n-1, c_{n}(i)=a_{n-2}(i)$ with $c_{n}(n-2)=a_{n-3}, b_{n}(i)=a_{n-1}(i)$ with $b_{n}(n-1)=a_{n-2}$, and

$$
a_{n}(i)=b_{n}(1)+\cdots+b_{n}(i)+c_{n}(i)
$$

and $a_{n}(n)=a_{n}(n-1)=a_{n-1}$ 
Lemma 10 shows

$$
a_{n}(i)=a_{n-1}(1)+\cdots+a_{n-1}(i)+a_{n-2}(i),
$$

with $a_{n}(n)=a_{n}(n-1)=a_{n-1}$ and $a_{n}(n-2)=a_{n-1}-a_{n-2}+a_{n-3}$. Since this is the same recurrence as in the previous case, we obtain the following result.

Theorem 11. Let $T=\{2314,1324,1243,1234\}$. Then

$$
F_{T}(x)=\frac{v\left(v(v-x)+(1+v) x^{2}\right)(1-v)}{x(v-x)},
$$

where $v$ is the root of $v=1+x-x^{2}-2 x / v+x^{2} / v^{2}$.

\subsection{Case 1054}

\subsubsection{The symmetry class of $\{2314,2431,2341,1342\}$}

Let $G_{m}(x)$ be the generating function for $T$-avoiders with $m$ left-right maxima. Clearly, $G_{0}(x)=$ 1 and $G_{1}(x)=x F_{T}(x)$.

Let us write an equation for $G_{2}(x)$. Let $\pi=i \pi^{\prime} n \pi^{\prime \prime} \in S_{n}(T)$ with exactly 2 left-right maxima. If $i=n-1$, then we have a contribution of $x\left(F_{T}(x)-1\right)$. So, we can assume that $i<n-1$, and then $\pi$ can be written as $\pi=i \pi^{\prime} n \alpha \beta$, where $\pi^{\prime} \alpha<i<\beta<n$ and $\beta \neq \emptyset$ and the contribution is $H(x)$. The contributions of the cases $\pi^{\prime} \alpha=\emptyset, \pi^{\prime}=(i-1) \pi^{\prime \prime \prime}, \pi^{\prime}=\pi^{\prime \prime}(i-1) \pi^{\prime \prime \prime}$ with $\pi^{\prime \prime} \neq \emptyset$, and $\alpha$ contains $i-1$ are given by $x^{2}(C(x)-1), x H(x), x^{3}(C(x)-1)^{2} C(x)$ and $x C(x) H(x)$ respectively. Thus, $G_{2}(x)=x\left(F_{T}(x)-1\right)+H(x)$, where

$$
H(x)=x^{2}(C(x)-1)+x H(x)+x^{3}(C(x)-1)^{2} C(x)+x C(x) H(x) .
$$

Hence,

$$
G_{2}(x)=x\left(F_{T}(x)-1\right)+\frac{x^{2}(C(x)-1)+x^{3}(C(x)-1)^{2} C(x)}{1-x-x C(x)} .
$$

Now let us write an equation for $G_{m}(x)$ with $m \geq 3$. Suppose $\pi=i_{1} \pi^{(1)} i_{2} \pi^{(2)} \cdots i_{m} \pi^{(m)} \in$ $S_{n}(T)$ has $m \geq 3$ left-right maxima. Since $\pi$ avoids $T$, we have $\pi^{(j)}>i_{j-1}$ for all $j$ (where $i_{0}:=0$ ). Thus, $\pi$ avoids $T$ if and only if $\pi^{(j)}$ avoids 231 for all $j$. Hence, $G_{m}(x)=x^{m} C^{m}(x)$ [3].

Summing over $m \geq 0$, we have

$$
F_{T}(x)=1+x F_{T}(x)+x\left(F_{T}(x)-1\right)+\frac{x^{2}(C(x)-1)+x^{3}(C(x)-1)^{2} C(x)}{1-x-x C(x)}+\frac{x^{3} C(x)^{3}}{1-x C(x)},
$$

and solving for $F_{T}(x)$ implies the following result.

Theorem 12. Let $T=\{2314,2431,2341,1342\}$. Then

$$
F_{T}(x)=C(x)+x^{3} C(x)^{5}+\frac{x^{4} C(x)^{5}}{1-2 x} .
$$




\subsubsection{The symmetry class of $\{2314,2341,1342,1243\}$}

Let $a_{n}=\left|S_{n}(T)\right|$ and define $a_{n}\left(i_{1}, i_{2}, \ldots, i_{s}\right)$ to be the number of permutations $\pi=i_{1} i_{2} \cdots i_{s} \pi^{\prime} \in$ $S_{n}(T)$.

Lemma 13. Let $n \geq 3$. Then $a_{n}(i, j)=0$ for $2 \leq i<j \leq n-1, a_{n}(1,2)=1$ and $a_{n}(1, i)=2^{i-3}$ for $i=3,4, \ldots, n-3, a_{n}(i, n)=a_{n-1}(i)$ for $i=1,2, \ldots, n-1, a_{n}(i, i-1)=a_{n-1}(i-1)$ for $i=2,3, \ldots, n, a_{n}(n)=a_{n}(n-1)=a_{n-1}, a_{n}(2,1)=2^{n-3}, a_{n}(i, 1)=a_{n-1}(i, 1)+2^{i-3}$ for $i=3,4, \ldots, n-2$, and

$$
a_{n}(i, j)=a_{n-1}(i, j)+\sum_{k=1}^{j-1} a_{n-1}(j, k)-a_{n-2}(j-1)+a_{n-1}(i-1, j-1),
$$

for $2 \leq j<i-1 \leq n-3$.

Proof. We consider the case $2 \leq j<i-1 \leq n-3$. By the definitions, we have

$$
a_{n}(i, j)=\sum_{k=1}^{j-2} a_{n}(i, j, k)+a_{n}(i, j, j-1)+\sum_{k=j+1}^{i-1} a_{n}(i, j, k)+\sum_{k=i+1}^{n-1} a_{n}(i, j, k)+a_{n}(i, j, n) .
$$

Clearly, $a_{n}(i, j, k)=a_{n-1}(j, k)$ and $a_{n}(i, j, j-1)=a_{n-1}(i-1, j-1)$ and $a_{n}(i, j, n)=a_{n-1}(i, j)$. Moreover, $a_{n}(i, j, k)=0$ for all $j+1 \leq k \leq n-1$ because we avoid 2314 and 2341 . Hence,

$$
a_{n}(i, j)=a_{n-1}(i, j)+\sum_{k=1}^{j-2} a_{n-1}(j, k)+a_{n-1}(i-1, j-1) .
$$

Since $a_{n}(j, j-1)=a_{n-1}(j-1)$ for $2 \leq j \leq n$, this completes the proof.

Define $a_{n}^{-}(i)=\sum_{j=1}^{i-1} a_{n}(i, j)$. By Lemma 13, we have

$$
\begin{aligned}
& a_{n}^{-}(i)-a_{n}(i, 1)-a_{n}(i, i-1) \\
& =a_{n-1}^{-}(i)-a_{n-1}(i, 1)-a_{n-1}(i, i-1)+\left(a_{n-1}^{-}(2)+\cdots+a_{n-1}^{-}(i-2)\right) \\
& -\left(a_{n-2}(1)+\cdots+a_{n-2}(i-3)\right)+a_{n-1}^{-}(i-1)-a_{n-2}(i-2),
\end{aligned}
$$

which is equivalent to

$$
a_{n}^{-}(i)=a_{n-1}^{-}(1)+\cdots+a_{n-1}^{-}(i)-\left(a_{n-2}(1)+\cdots+a_{n-2}(i-1)\right)+a_{n-1}(i-1)+2^{i-3},
$$

where $a_{n}^{-}(1)=0, a_{n}^{-}(2)=2^{n-3}$ and $a_{n}^{-}(n)=a_{n-1}$. Also, $a_{n}(i)=a_{n}^{-}(i)+a_{n-1}(i)+2^{n-3} \delta_{i=1}$ for all $i=1,2, \ldots, n$.

Define $A_{n}(v)=\sum_{i=1}^{n} a_{n}(i) v^{i-1}$ and $A_{n}^{-}(v)=\sum_{i=1}^{n} a_{n}^{-}(i) v^{i-1}$. Then, for all $n \geq 3$,

$$
A_{n}(v)=A_{n}^{-}(v)+A_{n-1}(v)+2^{n-3},
$$

and

$$
\begin{aligned}
A_{n}^{-}(v) & =v A_{n-1}(v)+\left(A_{n-1}(1)-A_{n-2}(1)\right) v^{n-1} \\
& +\frac{1}{1-v}\left(A_{n-1}^{-}(v)-v^{n-1} A_{n-1}^{-}(1)\right)-\frac{1}{1-v}\left(v A_{n-2}(v)-v^{n-1} A_{n-2}(1)\right)+v^{2} \sum_{i=0}^{n-4}(2 v)^{i} .
\end{aligned}
$$


with $A_{1}(v)=1, A_{2}(v)=1+v$ and $A_{2}^{-}(v)=v$.

Define $A(x, v)=\sum_{n \geq 0} A_{n}(v) x^{n}$ and $A^{-}(x, v)=\sum_{n \geq 0} A_{n}^{-}(v) x^{n}$. Then

$$
A(x, v)=\frac{1}{1-x}\left(1+\frac{x^{3}}{1-2 x}+A^{-}(x, v)\right),
$$

and

$$
\begin{aligned}
A^{-}(x, v) & =v x(A(x, v)-1)+x(A(v x, 1)-1)-v x^{2} A(v x, 1) \\
& +\frac{x}{1-v}\left(A^{-}(x, v)-A^{-}(v x, 1)\right)-\frac{v x^{2}}{1-v}(A(x, v)-A(v x, 1))+\frac{v^{2} x^{4}}{1-x-2 v x+2 v x^{2}} .
\end{aligned}
$$

Hence,

$$
\begin{aligned}
\frac{\left(v^{2}-v+x\right)(v x-v+x)}{v(1-v)(v-x)} A^{-}(x, v) & =\frac{x(v x-v+x)}{v(1-v)(1-x)} A^{-}(x, 1) \\
& +\frac{x^{2}}{v}+\frac{x^{4}\left(v^{3}-3 v^{2} x+2 v x^{2}-3 v x+2 x^{2}+v-x\right)}{v^{2}(1-2 x)(v-x)(v-2 x)} .
\end{aligned}
$$

By taking $v=1 / C(x)$, we obtain

$$
A^{-}(x, 1)=(1-x)\left(C(x)+x^{3} C(x)^{5}+\frac{x^{4} C(x)^{5}}{1-2 x}\right)-1-\frac{x^{3}}{1-2 x},
$$

which implies the following result.

Theorem 14. Let $T=\{2314,2341,1342,1243\}$. Then

$$
F_{T}(x)=C(x)+x^{3} C(x)^{5}+\frac{x^{4} C(x)^{5}}{1-2 x} .
$$

\subsubsection{The symmetry class of $\{1324,2341,1342,1234\}$}

Let $a_{n}=\left|S_{n}(T)\right|$ and define $a_{n}\left(i_{1}, i_{2}, \ldots, i_{s}\right)$ to be the number of permutations $\pi=i_{1} i_{2} \cdots i_{s} \pi^{\prime} \in$ $S_{n}(T)$.

Lemma 15. We have $a_{n}(i, j)=0$ for $2 \leq i+1<j \leq n-1, a_{n}(1,2)=1$ and $a_{n}(i, i+1)=2^{i-2}$ for $i=2,3, \ldots, n-2, a_{n}(i, n)=a_{n-1}(i)$ for $i=1,2, \ldots, n-1$, and $a_{n}(i, j)=a_{n-1}(j)$ for $1 \leq j<i \leq n$.

Proof. We prove only $a_{n}(i, j)=a_{n-1}(j)$ for $1 \leq j<i \leq n$, all the rest are done in the same fashion. For $\pi=i j \pi^{\prime} \in S_{n}(T)$, if there is an occurrence of 1324,1342 or 1234 that starts with $i$, then there is an occurrence of the same pattern that starts with $j$. Moreover, if there is an occurrence $i x y z$ of 2341 in $\pi$, then $j x y z$ is order isomorphic to 2341 or 1342 . Thus, $\pi$ avoids $T$ if and only if $j \pi^{\prime}$ avoids $T$, which implies that $a_{n}(i, j)=a_{n-1}(j)$ for $1 \leq j<i \leq n$.

By Lemma 15, we have

$$
a_{n}(i)=a_{n-1}(1)+a_{n-1}(2)+\cdots+a_{n-1}(i)+2^{i-2}
$$


with $a_{n}(n)=a_{n}(n-1)=a_{n-1}$ and $a_{n}(1)=1+a_{n-1}(1)$. Define $A_{n}(v)=\sum_{i=1}^{n-1} a_{n}(i) v^{i-1}$. Then,

$$
A_{n}(v)=\frac{1}{1-v}\left(A_{n-1}(v)-v^{n} A_{n-1}(1)\right)+1+v \frac{1-(2 v)^{n-3}}{1-2 v} .
$$

with $A_{0}(v)=A_{1}(v)=1$ and $A_{2}(v)=1+v$.

Define $A(x, v)=\sum_{n \geq 0} A_{n}(v) x^{n}$. Multiplying the recurrence for $A_{n}(v)$ by $x^{n} / v^{n}$ and summing over $n \geq 3$, we obtain

$$
A(x / v, v)=1+\frac{x}{v(1-v)}(A(x / v, v)-v A(x, 1))+\frac{x^{3}}{v^{2}(v-x)}+\frac{x^{4}}{v^{2}(v-x)(1-2 x)} .
$$

Taking $v=1 / C(x)$ and using the identity $A(x, 1)=F_{T}(x)$, we obtain the following result.

Theorem 16. Let $T=\{2314,2431,2341,1342\}$. Then

$$
F_{T}(x)=C(x)+x^{3} C(x)^{5}+\frac{x^{4} C(x)^{5}}{1-2 x} .
$$

\section{Proof of Theorem 2}

We treat the following selection of cases. Proofs for the others are similar and are omitted.

\subsection{Case 623}

\subsubsection{The symmetry class of $\{2413,3142,3241,1342\}$}

Clearly, $G_{0}(x)=1$ and $G_{1}(x)=x F_{T}(x)$. Let us write an equation for $G_{m}(x)$ with $m \geq$ 0 . Suppose $\pi=i_{1} \pi^{(1)} i_{2} \pi^{(2)} \cdots i_{m} \pi^{(m)} \in S_{n}(T)$ has $m$ left-right maxima. If $\pi^{(m)}>i_{m-1}$, then $\pi$ avoids $T$ if and only if $i_{1} \pi^{(1)} i_{2} \pi^{(2)} \cdots i_{m-1} \pi^{(m-1)}$ avoids $T$ and $\pi^{(m)}$ avoids 231, which implies the contribution of $x C(x) G_{m-1}(x)$ [3]. Otherwise, $\pi^{(m)}$ has a letter smaller than $i_{1}$, which implies that $\pi^{(j)}=\emptyset$ for all $j=1,2, \ldots, m$ and $\pi^{(m)}=\alpha \beta$ where $\alpha>i_{m-1}>i_{1}>\beta, \beta$ is not empty, and $\alpha$ avoids $\{213,231\}$ and $\beta$ avoids $T$. Thus, by [6], we have a contribution of $x^{m} \frac{1-x}{1-2 x}\left(F_{T}(x)-1\right)$. Hence,

$$
G_{m}(x)=x C(x) G_{m-1}(x)+x^{m} \frac{1-x}{1-2 x}\left(F_{T}(x)-1\right) .
$$

Summing over $m \geq 2$, we have

$$
F_{T}(x)=1+x F_{T}(x)+x C(x)\left(F_{T}(x)-1\right)+\frac{x^{2}}{1-2 x}\left(F_{T}(x)-1\right),
$$

and solving for $F_{T}(x)$ implies the following result.

Theorem 17. Let $T=\{2413,3142,3241,1342\}$. Then

$$
F_{T}(x)=\frac{x(2 x-1) C(x)-x^{2}-2 x+1}{x(2 x-1) C(x)+x^{2}-3 x+1} .
$$




\subsubsection{The symmetry class of $\{2431,1432,1324,1423\}$}

Note that each pattern in $T$ contains 132 . We recall the cell decomposition of $\pi \in S_{n}(T)$ as described in [4] (also, see [5]). If $\pi$ contains 132, then $\pi$ can be written as

$$
\pi=\alpha^{(1)} \cdots \alpha^{(j+2)} a \beta^{(1)}(a+1) \cdots \beta^{(i)}(a+i) \gamma(a+i+1) k_{1} \cdots k_{j},
$$

where $\alpha^{(1)}>k_{j+1}>\alpha^{(2)}>k_{j}>\cdots>\alpha^{(j+2)}>a+i+1, k_{j+1}$ is the maximal letter of $\gamma$, $a k_{j+1}(a+i+1)$ is order isomorphic to $132, \beta^{(1)}>\cdots>\beta^{(i)}>\gamma^{\prime}$ where $\gamma^{\prime}$ is obtained from $\gamma$ by removing the letter $k_{j+1}$ and each of $\alpha^{(s)}, \beta^{(s)}$ and $\gamma$ avoids 132 . Hence, by [3], we have

$$
F_{T}(x)=C(x)+\frac{x^{2} C(x)^{2}(C(x)-1)}{(1-x C(x))^{2}},
$$

which leads to the following result.

Theorem 18. Let $T=\{2431,1432,1324,1423\}$. Then

$$
F_{T}(x)=\frac{x(2 x-1) C(x)-x^{2}-2 x+1}{x(2 x-1) C(x)+x^{2}-3 x+1} .
$$

\subsection{Case 651}

\subsubsection{The symmetry class of $\{3142,2431,1423,1324\}$}

Clearly, $G_{0}(x)=1$ and $G_{1}(x)=x F_{T}(x)$. Let us write an equation for $G_{m}(x)$ with $m \geq 2$. Suppose $\pi=i_{1} \pi^{(1)} i_{2} \pi^{(2)} \cdots i_{m} \pi^{(m)} \in S_{n}(T)$ has $m \geq 2$ left-right maxima. Since $\pi$ avoids 1324 , we have that $\pi^{(j)}<i_{1}$ for all $j=1,2, \ldots, m-1$. Since $\pi$ avoids 3142 and $2431, \pi^{(m)}$ can be written as $\alpha^{(1)} \alpha^{(2)} \cdots \alpha^{(m)}$ where $i_{j-1}<\alpha^{(j)}<i_{j}$ with $i_{0}=0$. Thus, $\pi$ avoids $T$ if and only if either

- there exists $j, 2 \leq j \leq m$, such that $\pi^{(1)}>\cdots>\pi^{(j-1)}>\alpha^{(1)}, \alpha^{(j)}$ is decreasing and $\alpha^{(s)}=\emptyset$ for all $s=2, \ldots, j-1, j+1, \ldots, m$, and $\pi^{(s)}$ avoids 132 for all $s=1,2, \ldots, j-1$, and $\alpha^{(1)}$ avoids 132 , or

- $\pi^{(1)}>\cdots>\pi^{(m-1)}>\alpha^{(1)}, \alpha^{(s)}=\emptyset$ for all $s=2,3, \ldots, m$, and $\pi^{(s)}$ avoids 132 for all $s=1,2, \ldots, m-1$, and $\alpha^{(1)}$ avoids $T$.

Thus, by [3], we have

$$
G_{m}(x)=\sum_{j=2}^{m} x^{m} C(x)^{j} \frac{x}{1-x}+x^{m} C(x)^{m-1} F_{T}(x) .
$$

Hence, since $F_{T}(x)=1+x F_{T}(x)+\sum_{m \geq 2} G_{m}(x)$, we obtain the following result.

Theorem 19. Let $T=\{3142,2431,1423,1324\}$. Then

$$
F_{T}(x)=\frac{\left(1-2 x-x\left(x^{2}-3 x+1\right) C(x)\right) C(x)^{2}}{(1-x)^{2}}
$$




\subsection{Case 729}

\subsubsection{The symmetry class of $\{3142,1324,1423,1243\}$}

Let $T=\{3142,1243,1423,1324\}$. Clearly, $G_{0}(x)=1$ and $G_{1}(x)=x F_{T}(x)$. Let us write an equation for $G_{m}(x)$ with $m \geq 2$. Suppose $\pi=i_{1} \pi^{(1)} i_{2} \pi^{(2)} \cdots i_{m} \pi^{(m)} \in S_{n}(T)$ has $m \geq 2$ leftright maxima. Since $\pi$ avoids 1324 and 1243, we have that $\pi^{(j)}<i_{1}$ for all $j=1,2, \ldots, m-1$ and $\pi^{(m)}<i_{2}$. Since $\pi$ avoids $1423, \pi^{(m)}$ contains the subsequence $\left(i_{2}-1\right)\left(i_{2}-2\right) \cdots\left(i_{1}+1\right)$. So $\pi^{(m)}$ has the form $\alpha^{(1)}\left(i_{2}-1\right) \cdots \alpha^{\left(i_{2}-i_{1}-1\right)}\left(i_{1}+1\right) \alpha^{\left(i_{2}-i_{1}\right)}$, and $\pi$ avoids $T$ if and only if either

- $\pi^{(1)}>\cdots>\pi^{(m)}, \pi^{(s)}$ avoids 132 for all $s=1,2, \ldots, m-1$, and $\pi^{(m)}$ avoids $T$ (case $\left.i_{2}=i_{1}+1\right)$.

- $\pi^{(1)} \cdots \pi^{(m-1)} \alpha^{(1)} \cdots \alpha^{\left(i_{2}-i_{1}-2\right)}$ is decreasing, $\pi^{(2)} \cdots \pi^{(m-1)}=\emptyset, \alpha^{\left(i_{2}-i_{1}-1\right)}$ avoids 132 , and $\alpha^{\left(i_{2}-i_{1}\right)}$ avoids $T$ (case $i_{2}>i_{1}+1$ ).

Thus, by [3], we have

$$
G_{m}(x)=x^{m} C(x)^{m-1} F_{T}(x)+\frac{x^{m+1} C(x) F_{T}(x)}{1-2 x} .
$$

Since $F_{T}(x)=1+x F_{T}(x)+\sum_{m \geq 2} G_{m}(x)$, we obtain the following result.

Theorem 20. Let $T=\{3142,1243,1423,1324\}$. Then

$$
F_{T}(x)=\frac{(1-x)(1-2 x)}{(1-x)(1-2 x)-x\left(1-3 x+3 x^{2}\right) C(x)} .
$$

\section{References}

[1] Arıkan, T., Kılıç, E., \& Mansour, T. (2017) A Wilf class composed of 19 symmetry classes of quadruples of 4-letter patterns, Notes on Number Theory and Discrete Mathematics, 23(3), 79-99.

[2] Callan, D. \& Mansour, T. (2017) Enumeration of 2-Wilf classes of four 4-letter patterns, Turkish Journal of Analysis and Number Theory, 5(6), 210-225.

[3] Knuth, D. E. (1997) The Art of Computer Programming, 3rd edition, Addison Wesley, Reading, MA.

[4] Mansour, T. \& Schork, M. (2018) Permutation patterns and cell decompositions, Mathematics in Computer Science, to appear, https://doi.org/10.1007/s11786-018-0353-5.

[5] Mansour, T. \& Vainshtein, A. (2001) Restricted 132-avoiding permutations, Advances in Applied Mathematics, 26, 258-269.

[6] Simion, R. \& Schmidt, F. W. (1985) Restricted permutations, European Journal of Combinatorics, 6, 383-406.

[7] Vatter, V. (2012) Finding regular insertion encodings for permutation classes, Journal of Symbolic Computation, 47(3), 259-265. 


\section{Appendix}

Table 1. 3-Wilf classes of four 4-letter patterns

\begin{tabular}{|c|c|c|c|}
\hline No. & Pattern set $T$ & Generating function $F_{T}(x)$ & Thm./[Ref] \\
\hline 75 & $\begin{array}{l}\{4231,2341,4312,4123\} \\
\{2314,3124,1432,1324\} \\
\{2413,3142,1423,1234\}\end{array}$ & $\frac{(1-x)^{4}\left(1-3 x+2 x^{2}-x^{3}\right)}{x^{8}-5 x^{7}+17 x^{6}-36 x^{5}+52 x^{4}-47 x^{3}+26 x^{2}-8 x+1}$ & $\begin{array}{l}\text { INSENC } \\
\text { INSENC } \\
\text { INSENC }\end{array}$ \\
\hline 141 & $\begin{array}{l}\{4231,3412,1423,1234\} \\
\{2314,4312,1342,1324\} \\
\{2134,4312,3124,1342\}\end{array}$ & $\frac{9 x^{4}-11 x^{3}+11 x^{2}-5 x+1}{(1-x)^{6}}$ & $\begin{array}{l}\text { INSENC } \\
\text { INSENC } \\
\text { INSENC }\end{array}$ \\
\hline 212 & $\begin{array}{l}\{2431,4213,1432,1324\} \\
\{3412,3124,1342,4123\} \\
\{3412,3124,1342,1423\}\end{array}$ & $\frac{1-6 x-15 x^{3}-2 x^{5}+9 x^{4}+14 x^{2}}{\left(x^{2}-3 x+1\right)(1-x)^{4}}$ & $\begin{array}{c}\text { INSENC } \\
\text { EX } \\
\text { EX }\end{array}$ \\
\hline 217 & $\begin{array}{l}\{2431,4213,1324,1423\} \\
\{2413,4132,1243,1234\} \\
\{2143,3412,1342,1423\}\end{array}$ & $\frac{3 x^{4}-6 x^{3}+9 x^{2}-5 x+1}{\left(x^{2}-3 x+1\right)(1-x)^{3}}$ & $\begin{array}{l}\text { INSENC } \\
\text { INSENC } \\
\text { EX }\end{array}$ \\
\hline 231 & $\begin{array}{l}\{2431,2134,4312,1423\} \\
\{2413,4312,1342,1234\} \\
\{3412,4132,1243,1234\}\end{array}$ & $\frac{x^{7}-2 x^{6}-x^{5}+9 x^{4}-11 x^{3}+11 x^{2}-5 x+1}{(1-x)^{6}}$ & $\begin{array}{l}\text { INSENC } \\
\text { INSENC } \\
\text { INSENC }\end{array}$ \\
\hline 301 & $\begin{array}{l}\{2341,2143,4132,4123\} \\
\{2314,4213,3412,1432\} \\
\{3412,1432,1324,4123\}\end{array}$ & $\frac{x^{6}-7 x^{5}+13 x^{4}-18 x^{3}+15 x^{2}-6 x+1}{(1-2 x)(1-x)^{5}}$ & $\begin{array}{l}\text { INSENC } \\
\text { INSENC } \\
\text { INSENC }\end{array}$ \\
\hline 354 & $\begin{array}{l}\{2341,3412,4123,1243\} \\
\{3412,2341,1324,1234\} \\
\{3412,1324,1243,1234\}\end{array}$ & $\frac{1-7 x+21 x^{2}-33 x^{3}+31 x^{4}-16 x^{5}+3 x^{6}-2 x^{7}}{(1-2 x)(1-x)^{6}}$ & $\begin{array}{l}\text { EX } \\
\text { EX } \\
\text { EX }\end{array}$ \\
\hline 482 & $\begin{array}{l}\{4213,2143,3142,1342\} \\
\{3142,1342,4123,1243\} \\
\{4132,1432,4123,1243\}\end{array}$ & $\frac{(1-2 x)\left(3 x^{4}-7 x^{3}+9 x^{2}-5 x+1\right.}{(1-x)^{5}(1-3 x)}$ & $\begin{array}{l}\text { INSENC } \\
\text { INSENC } \\
\text { INSENC }\end{array}$ \\
\hline 538 & $\begin{array}{l}\{4213,3142,1342,1243\} \\
\{2143,3142,1342,4123\} \\
\{3412,1432,4123,1423\}\end{array}$ & $\frac{-\left(4 x^{4}-7 x^{3}+9 x^{2}-5 x+1\right)}{\left(2 x^{3}-4 x^{2}+4 x-1\right)(1-x)^{2}}$ & $\begin{array}{l}\text { INSENC } \\
\text { INSENC } \\
\text { INSENC }\end{array}$ \\
\hline 548 & $\begin{array}{l}\{4213,3124,1342,1423\} \\
\{2143,3142,1324,4123\} \\
\{3142,3124,1432,4123\}\end{array}$ & $\frac{x^{5}-3 x^{4}+4 x^{3}-8 x^{2}+5 x-1}{\left(x^{3}-2 x^{2}+3 x-1\right)\left(x^{2}-3 x+1\right)}$ & $\begin{array}{l}\text { INSENC } \\
\text { EX } \\
\text { INSENC }\end{array}$ \\
\hline 602 & $\begin{array}{l}\{2413,4312,3412,1342\} \\
\{2413,4312,3142,1342\} \\
\{2413,3412,4132,1342\}\end{array}$ & $\frac{x^{5}-5 x^{4}+16 x^{3}-17 x^{2}+7 x-1}{(3 x-1)\left(x^{2}-3 x+1\right)(1-x)^{2}}$ & $\begin{array}{l}\text { INSENC } \\
\text { INSENC } \\
\text { EX }\end{array}$ \\
\hline 609 & $\begin{array}{l}\{2413,4312,1432,1324\} \\
\{2413,4312,1342,1324\} \\
\{2143,4312,3142,1324\}\end{array}$ & $\frac{x^{6}-9 x^{5}+21 x^{4}-28 x^{3}+20 x^{2}-7 x+1}{(1-2 x)^{2}(1-x)^{4}}$ & $\begin{array}{l}\text { INSENC } \\
\text { INSENC } \\
\text { EX }\end{array}$ \\
\hline 614 & $\begin{array}{l}\{2413,3412,3142,1234\} \\
\{2134,4312,4132,1432\} \\
\{2134,4312,1342,1324\}\end{array}$ & $\frac{x^{6}-8 x^{5}+20 x^{4}-22 x^{3}+16 x^{2}-6 x+1}{(1-x)^{7}}$ & $\begin{array}{l}\text { INSENC } \\
\text { INSENC } \\
\text { INSENC }\end{array}$ \\
\hline
\end{tabular}




\begin{tabular}{|c|c|c|c|}
\hline No. & Pattern set $T$ & Generating function $F_{T}(x)$ & Thm./[Ref] \\
\hline 619 & $\begin{array}{l}\{2413,3412,4132,1234\} \\
\{2134,4312,3412,1342\} \\
\{2134,3412,4132,1342\}\end{array}$ & $\frac{2 x^{6}-10 x^{5}+20 x^{4}-22 x^{3}+16 x^{2}-6 x+1}{(1-x)^{7}}$ & $\begin{array}{l}\text { INSENC } \\
\text { INSENC } \\
\text { INSENC }\end{array}$ \\
\hline 643 & $\begin{array}{l}\{2413,3124,1432,1324\} \\
\{2143,3142,3124,1432\} \\
\{3142,3124,1423,1234\}\end{array}$ & $\frac{(1-x)^{3}(2 x-1)}{x^{5}-6 x^{4}+14 x^{3}-13 x^{2}+6 x-1}$ & $\begin{array}{l}\text { INSENC } \\
\text { INSENC } \\
\text { INSENC }\end{array}$ \\
\hline 656 & $\begin{array}{l}\{2431,3142,1324,1423\} \\
\{2431,2413,1324,1423\} \\
\{2341,2314,1243,1234\}\end{array}$ & $C(x)+\frac{x^{3}}{1-2 x} C(x)^{4}$ & $\begin{array}{c}\text { [4] } \\
\text { [4] } \\
\text { Thm. } 5\end{array}$ \\
\hline 686 & $\begin{array}{l}\{2143,4312,3124,1243\} \\
\{4312,3124,1432,1243\} \\
\{4312,1432,1324,4123\}\end{array}$ & $\frac{x^{7}-10 x^{5}+21 x^{4}-28 x^{3}+20 x^{2}-7 x+1}{(x-1)^{4}(2 x-1)^{2}}$ & $\begin{array}{l}\text { INSENC } \\
\text { INSENC } \\
\text { INSENC }\end{array}$ \\
\hline 702 & $\begin{array}{l}\{2143,3412,3142,1342\} \\
\{3412,3142,1342,1324\} \\
\{3412,3142,1342,1243\}\end{array}$ & $\frac{1-6 x+11 x^{2}-5 x^{3}}{(1-x)(1-3 x)\left(1-3 x+x^{2}\right)}$ & $\begin{array}{l}\text { EX } \\
\text { EX } \\
\text { EX }\end{array}$ \\
\hline 721 & $\begin{array}{l}\{2143,3412,1423,1243\} \\
\{4312,3142,3124,1342\} \\
\{3124,4132,1342,1423\}\end{array}$ & $\frac{x^{6}-4 x^{5}+10 x^{4}-20 x^{3}+18 x^{2}-7 x+1}{\left(x^{2}-3 x+1\right)^{2}(x-1)^{2}}$ & $\begin{array}{c}\text { EX } \\
\text { INSENC } \\
\text { INSENC }\end{array}$ \\
\hline 726 & $\begin{array}{l}\{2143,3142,1432,1423\} \\
\{2143,3142,1342,1423\} \\
\{3142,1432,1423,1243\}\end{array}$ & $\frac{\left(1-x-\sqrt{1-6 x+9 x^{2}-8 x^{3}}\right)(1-x)}{2 x\left(1-2 x+2 x^{2}\right)}$ & $\begin{array}{l}{[4]} \\
{[4]} \\
{[4]}\end{array}$ \\
\hline 733 & $\begin{array}{l}\{2143,3142,1423,1234\} \\
\{3142,1432,1243,1234\} \\
\{3124,1432,1342,1234\}\end{array}$ & $\frac{x^{3}-2 x^{2}+3 x-1}{x^{4}+3 x^{3}-4 x^{2}+4 x-1}$ & $\begin{array}{l}\text { INSENC } \\
\text { INSENC } \\
\text { INSENC }\end{array}$ \\
\hline 824 & $\begin{array}{l}\{2134,3142,1432,1342\} \\
\{2134,3142,1432,1423\} \\
\{3142,1432,1342,1234\}\end{array}$ & $\frac{\left(x^{3}-2 x^{2}+3 x-1\right)^{2}}{3 x^{6}-9 x^{5}+20 x^{4}-24 x^{3}+18 x^{2}-7 x+1}$ & $\begin{array}{l}\text { INSENC } \\
\text { INSENC } \\
\text { INSENC }\end{array}$ \\
\hline 845 & $\begin{array}{l}\{2134,4132,1432,1324\} \\
\{3124,4132,1423,1243\} \\
\{4132,1432,1324,4123\}\end{array}$ & $-\frac{\left(x^{5}-7 x^{4}+19 x^{3}-18 x^{2}+7 x-1\right)}{(1-x)\left(x^{2}-3 x+1\right)(1-2 x)^{2}}$ & $\begin{array}{l}\text { INSENC } \\
\text { INSENC } \\
\text { INSENC }\end{array}$ \\
\hline 882 & $\begin{array}{l}\{4312,3412,3142,4123\} \\
\{2143,3142,1342,1432\} \\
\{3142,1432,1342,1243\}\end{array}$ & $f=1-x+x^{2} f+x\left(x^{2}-2 x+2\right) f^{2}-x^{2}(1-x) f^{3}$ & $\begin{array}{l}{[4]} \\
{[4]} \\
{[4]}\end{array}$ \\
\hline 890 & $\begin{array}{l}\{2143,1243,1423,1432\} \\
\{2413,1432,1423,1342\} \\
\{2314,1324,1243,1234\}\end{array}$ & $\begin{array}{c}\frac{v\left(v(v-x)+(1+v) x^{2}\right)(1-v)}{x(v-x)} \\
\text { where } v=1+x-x^{2}-2 x / v+x^{2} / v^{2}\end{array}$ & $\begin{array}{l}\text { Thm. } 7 \\
\text { Thm. } 9 \\
\text { Thm. } 11 \\
\end{array}$ \\
\hline 1034 & $\begin{array}{l}\{3142,3124,1432,1342\} \\
\{3142,1324,1243,1234\} \\
\{3124,1432,1342,1423\}\end{array}$ & $\frac{(1-2 x)\left(x^{2}+2 x-1\right)}{\left(2 x^{3}+2 x^{2}-4 x+1\right)(x-1)}$ & $\begin{array}{l}\text { INSENC } \\
\text { INSENC } \\
\text { INSENC }\end{array}$ \\
\hline 1054 & $\begin{array}{l}\{2314,2431,2341,1342\} \\
\{2314,2341,1342,1243\} \\
\{1324,2341,1342,1234\}\end{array}$ & $C(x)+x^{3} C(x)^{5}+\frac{x^{4} C(x)^{5}}{1-2 x}$ & $\begin{array}{l}\text { Thm. } 12 \\
\text { Thm. } 14 \\
\text { Thm. } 16\end{array}$ \\
\hline
\end{tabular}


Table 2. 4-Wilf classes of four 4-letter patterns

\begin{tabular}{|c|c|c|c|}
\hline No. & Pattern set $T$ & Generating function $F_{T}(x)$ & Thm./[Ref] \\
\hline 74 & $\begin{array}{l}\{4231,2341,2143,4123\} \\
\{4231,3412,1243,1234\} \\
\{2143,3412,1324,1234\} \\
\{2143,3412,4123,1234\}\end{array}$ & $\frac{x^{6}-2 x^{5}-5 x^{4}+4 x^{3}-7 x^{2}+4 x-1}{(x-1)^{5}}$ & $\begin{array}{l}\text { INSENC } \\
\text { INSENC } \\
\text { INSENC } \\
\text { INSENC }\end{array}$ \\
\hline 132 & $\begin{array}{l}\{4231,3412,3142,1234\} \\
\{2143,3412,3142,1234\} \\
\{2143,3412,1243,1234\} \\
\{2134,4312,3142,1432\}\end{array}$ & $\frac{-\left(x^{5}-9 x^{4}+11 x^{3}-11 x^{2}+5 x-1\right)}{(x-1)^{6}}$ & $\begin{array}{l}\text { INSENC } \\
\text { INSENC } \\
\text { INSENC } \\
\text { INSENC }\end{array}$ \\
\hline 156 & $\begin{array}{l}\{4231,3124,1342,1324\} \\
\{4231,1342,1324,1423\} \\
\{3412,3124,1324,1423\} \\
\{3412,1324,4123,1423\}\end{array}$ & $\frac{-\left(6 x^{5}-21 x^{4}+28 x^{3}-20 x^{2}+7 x-1\right)}{(2 x-1)^{2}(x-1)^{4}}$ & $\begin{array}{l}\text { INSENC } \\
\text { EX } \\
\text { EX } \\
\text { EX }\end{array}$ \\
\hline 163 & $\begin{array}{l}\{4231,4132,1342,1324\} \\
\{4312,3124,4132,1324\} \\
\{3412,3142,3124,1243\} \\
\{3412,3142,1342,1234\}\end{array}$ & $\frac{-\left(13 x^{5}-35 x^{4}+42 x^{3}-26 x^{2}+8 x-1\right)}{(2 x-1)^{3}(x-1)^{3}}$ & $\begin{array}{c}\text { EX } \\
\text { EX } \\
\text { INSENC } \\
\text { INSENC }\end{array}$ \\
\hline 361 & $\begin{array}{l}\{2341,3142,4123,1423\} \\
\{4213,2413,3124,1432\} \\
\{4213,3142,3124,1432\} \\
\{4213,3142,1432,1324\}\end{array}$ & $\frac{x^{5}-6 x^{4}+12 x^{3}-13 x^{2}+6 x-1}{\left(3 x^{3}-5 x^{2}+4 x-1\right)\left(x^{2}-3 x+1\right)}$ & $\begin{array}{l}\text { INSENC } \\
\text { INSENC } \\
\text { INSENC } \\
\text { INSENC }\end{array}$ \\
\hline 375 & $\begin{array}{l}\{2341,4132,1432,1324\} \\
\{4213,3124,4132,1342\} \\
\{2143,3412,1432,1243\} \\
\{2143,1324,4123,1234\}\end{array}$ & $\frac{-\left(2 x^{4}+x^{3}+4 x^{2}-4 x+1\right)}{(2 x-1)\left(x^{2}-3 x+1\right)}$ & $\begin{array}{l}\text { INSENC } \\
\text { INSENC } \\
\text { EX } \\
\text { INSENC }\end{array}$ \\
\hline 534 & $\begin{array}{l}\{4213,3142,4132,1324\} \\
\{3142,3124,4132,1324\} \\
\{3142,3124,1324,4123\} \\
\{1432,1324,4123,1423\}\end{array}$ & $\frac{-\left(3 x^{4}-15 x^{3}+17 x^{2}-7 x+1\right)}{(2 x-1)\left(x^{2}-3 x+1\right)^{2}}$ & $\begin{array}{c}\text { EX } \\
\text { EX } \\
\text { EX } \\
\text { INSENC }\end{array}$ \\
\hline 623 & $\begin{array}{l}\{2413,3142,3241,1342\} \\
\{2431,1432,1324,1423\} \\
\{4132,1432,1324,1243\} \\
\{1342,4123,1423,1234\}\end{array}$ & $\frac{\left.x(2 x-1) C(x)-x^{2}-2 x+1\right)}{x(2 x-1) C(x)+x^{2}-3 x+1}$ & $\begin{array}{c}\text { Theorem } 17 \\
\text { Theorem } 18 \\
\text { EX } \\
\text { EX }\end{array}$ \\
\hline 647 & $\begin{array}{l}\{2413,3124,1342,1324\} \\
\{2134,3142,3124,1423\} \\
\{3142,3124,1324,1423\} \\
\{3124,1432,1324,1423\}\end{array}$ & $\frac{-(2 x-1)^{3}}{5 x^{4}-17 x^{3}+17 x^{2}-7 x+1}$ & $\begin{array}{c}\text { EX } \\
\text { EX } \\
\text { EX } \\
\text { INSENC }\end{array}$ \\
\hline 651 & $\begin{array}{l}\{2413,4132,1432,1324\} \\
\{3142,2431,1423,1324\} \\
\{2143,4132,1432,1324\}\end{array}$ & $\frac{\left(1-2 x-x\left(x^{2}-3 x+1\right) C(x)\right) C^{2}(x)}{(1-x)^{2}}$ & $\begin{array}{c}\text { EX } \\
\text { Theorem } 19 \\
\text { EX } \\
\end{array}$ \\
\hline
\end{tabular}




\begin{tabular}{|c|c|c|c|}
\hline No. & Pattern set $T$ & Generating function $F_{T}(x)$ & Thm./[Ref] \\
\hline & $\{3142,4132,1432,1243\}$ & & EX \\
\hline 659 & $\{2413,4132,4123,1234\}$ & & INSENC \\
& $\{2134,3412,3142,1423\}$ & & EX \\
& $\{3412,1342,4123,1243\}$ & $\frac{-\left(7 x^{4}-14 x^{3}+14 x^{2}-6 x+1\right)}{(x-1)^{3}(2 x-1)^{2}}$ & EX \\
& $\{3412,1342,4123,1234\}$ & & EX \\
\hline 670 & $\{2143,2134,4132,1342\}$ & & INSENC \\
& $\{2143,3412,1432,1423\}$ & & EX \\
& $\{3412,3142,1432,1324\}$ & $\frac{2 x^{5}-4 x^{4}+11 x^{3}-13 x^{2}+6 x-1}{(2 x-1)\left(x^{2}-3 x+1\right)(x-1)^{2}}$ & EX \\
& $\{3412,3142,1432,1243\}$ & & EX \\
\hline 729 & $\{2143,3142,1324,1423\}$ & & EX \\
& $\{3412,3142,4123,1423\}$ & & EX \\
& $\{3142,1324,1423,1243\}$ & $\frac{(1-x)(1-2 x)}{(1-x)(1-2 x)-x\left(1-3 x+3 x^{2}\right) C(x)}$ & Theorem 20 \\
& $\{3124,1342,1423,1243\}$ & & EX \\
\hline 769 & $\{2143,1342,4123,1423\}$ & & INSENC \\
& $\{2143,1342,4123,1243\}$ & & INSENC \\
& $\{3412,4132,1432,4123\}$ & $\frac{x^{4}-x^{3}+4 x^{2}-4 x+1}{(x-1)\left(x^{3}-3 x^{2}+4 x-1\right)}$ & INSENC \\
& $\{1432,1342,4123,1243\}$ & & INSENC \\
\hline 778 & $\{2134,4312,3142,1342\}$ & & INSENC \\
& $\{4312,3412,1342,1234\}$ & & INSENC \\
& $\{3412,3124,4132,1234\}$ & $\frac{-\left(2 x^{6}-9 x^{5}+20 x^{4}-22 x^{3}+16 x^{2}-6 x+1\right)}{(x-1)^{7}}$ & INSENC \\
& $\{3412,4132,1342,1234\}$ & & INSENC \\
\hline
\end{tabular}

